\title{
Infinite distance and zero gauge coupling in 5D supergravity
}

\author{
Ben Heidenreich $\oplus^{*}$ \\ Department of Physics, University of Massachusetts, Amherst, Massachusetts 01003, USA \\ Tom Rudelius $\oplus^{\dagger}$ \\ School of Natural Sciences, Institute for Advanced Study, Princeton, New Jersey 08540, USA \\ and Department of Physics, University of California, Berkeley, California 94720, USA
}

(Received 30 August 2020; accepted 27 October 2021; published 29 November 2021)

\begin{abstract}
In this article, we prove that for all five-dimensional supergravities arising from $\mathrm{M}$ theory compactified on a Calabi-Yau threefold, points of vanishing gauge coupling lie at infinite distance in the moduli space. Conversely, any point at infinite distance in the vector multiplet moduli space is a point of vanishing gauge coupling. This agrees with expectations from the tower/sublattice weak gravity conjecture, the swampland distance conjecture, and the emergence proposal.
\end{abstract}

DOI: 10.1103/PhysRevD.104.106016

\section{INTRODUCTION}

Quantum gravities with sufficient supersymmetry tend to have (i) gauge fields and (ii) continuous moduli spaces of vacua controlled by expectation values of scalar fields [1]. Recently, much work has gone into understanding the universal behavior that arises in weakly coupled limits of these gauge theories and in asymptotic, infinite distance limits of these moduli spaces. This had led to a number of "swampland conjectures" that attempt to describe this universal behavior, along with other general features of quantum gravities. Among them are the tower/sublattice weak gravity conjecture (T/sLWGC) [2-5], the swampland distance conjecture (SDC) [1], and the emergence proposal (EP) [6-9].

The T/sLWGC requires an infinite tower of particles to become massless at a point of vanishing gauge coupling, whereas the SDC requires an infinite tower of particles to become massless at an infinite distance point in moduli space. In turn, the EP suggests that a vanishing gauge coupling should emerge as an infinite tower of charged particles become light [7], and infinite scalar field distance should emerge as an infinite tower of particles become light $[8,9]$.

Thus, a supergravity theory satisfying the T/sLWGC will have an infinite tower of massless charged particles in the limit of vanishing gauge coupling, which, by the EP, should

\footnotetext{
*bheidenreich@umass.edu

†rudelius@berkeley.edu
}

Published by the American Physical Society under the terms of the Creative Commons Attribution 4.0 International license. Further distribution of this work must maintain attribution to the author(s) and the published article's title, journal citation, and DOI. Funded by SCOAP . generate an infinite distance in scalar field space. Conversely, a theory satisfying the SDC will have an infinite tower of massless particles in the limit of infinite distance, which, by the EP, should generate a vanishing gauge coupling (assuming the particles are charged).

In this article, we will see that these expectations are borne out in 5D supergravity theories arising from M-theory compactifications on Calabi-Yau threefolds: points of vanishing gauge coupling are at infinite distance, and points at infinite distance in vector multiplet moduli space have vanishing gauge coupling. ${ }^{1}$ These results complement similar results in 4D/5D supergravity theories arising from type II/M-theory compactifications on CalabiYau threefolds $[8,11,12]$ and $6 \mathrm{D}$ supergravity theories arising from F-theory compactifications on elliptically fibered Calabi-Yau threefolds $[10,13,14]$.

The remainder of the article is structured as follows. We begin with a brief review of relevant properties of CalabiYau threefolds and 5D supergravity, and we derive a related positivity lemma. We then prove that points of zero gauge coupling lie at infinite distance in the moduli space as well as the converse statement.

\section{THE KÄHLER CONE OF A CALABI-YAU THREEFOLD}

A Calabi-Yau threefold $X$ is equipped with a $(1,1)$-form $J$ known as the Kähler form, which takes values inside a strongly convex polyhedral cone whose interior is the Kähler cone, $\mathcal{K}(X)$. Within the Kähler cone, $J$ can be expressed as a positive linear combination,

\footnotetext{
${ }^{1}$ These points of zero gauge coupling and infinite distance are associated geometrically with curves or divisors of the CalabiYau going to infinite size [10].
} 


$$
J=\sum_{i} \omega_{i} t^{i}, \quad t^{i}>0,
$$

where each $\omega_{i}, i=1, \ldots, N$ is called a generator of the Kähler cone. If the number of generators $N$ is equal to the dimension $h^{1,1}(X)$ of the Kähler cone, the cone is simplicial. If $N>h^{1,1}(X)$, the cone is nonsimplicial.

Any $(1,1)$-form $\lambda$ in the closure of the Kähler cone is called nef. Nef $(1,1)$-forms have the property that their triple product is non-negative: $\lambda_{1} \cdot \lambda_{2} \cdot \lambda_{3} \geq 0$ for $\lambda_{i}$ nef. In particular, this implies that the triple intersection of any three generators of the Kähler cone is non-negative.

If the Kähler cone is simplicial, we can take the generators $\omega_{I}$ of the Kähler cone to be a basis of $h^{1,1}(X)$. Thus, we can write $J=\sum_{I} \omega_{I} Y^{I}$, with $Y^{I}>0$, and the triple intersection numbers are all non-negative:

$$
C_{I J K}:=\omega_{I} \cdot \omega_{J} \cdot \omega_{K} \geq 0, I, J, \quad K=1, \ldots, h^{1,1}(X) .
$$

Such a basis does not exist when the Kähler cone is nonsimplicial [15]. One can choose a subset of the generators as a basis for $h^{1,1}(X)$, but then one cannot express $J$ as a positive linear combination of these particular generators over the entirety of the Kähler cone.

In addition, the Kähler form $J$ is often able to cross certain codimension-1 boundaries of the Kähler cone $\mathcal{K}(X)$ into the Kähler cone $\mathcal{K}(\tilde{X})$ of a birationally equivalent Calabi-Yau $\tilde{X}$, which is related to $X$ by a flop transition. The union of the Kähler cones of all of these birationally equivalent Calabi-Yau threefolds is called the extended Kähler cone of $X$, which we denote $\mathcal{K}_{\cup}(X)$. Within a given Kähler cone $\mathcal{K}(X), J$ may be written as $J=\sum_{I} \omega_{I} Y^{I}$, with $Y^{I}>0$ and $C_{I J K} \geq 0$, but this positivity will cease to hold as $J$ passes through the boundary between the two Kähler cones, as some $Y^{I}$ switches from positive to negative.

These subtleties are avoided if $J$ is contained in a particular, simplicial subcone of a particular Kähler cone $\mathcal{K}(X)$. In particular, consider generic paths in the extended Kähler cone of some Calabi-Yau $X$,

$$
s \gamma:[0,1] \rightarrow \mathcal{K}_{\cup}(X), \quad x \mapsto J(x),
$$

where $J(x)$ approaches either an asymptotic boundary or a point of zero gauge coupling as $x \rightarrow 0$. If $x_{0}>0$ exists such that for $0 \leq x \leq x_{0}, J(x)$ is contained within the closure of a particular simplicial subcone of a single Kähler cone $\mathcal{K}(X)$ with generators $\left\{\omega_{I}\right\}$, then we may write

$J(x)=\sum_{I=1}^{h^{1,1}(X)} \omega_{I} Y^{I}(x), \quad Y^{I}(x) \geq 0 \quad$ for all $x \in\left[0, x_{0}\right]$.

Notably, the Kähler moduli $Y^{I}$ depend on $x$, but the basis $(1,1)$-forms $\omega_{I}$ do not. In this basis, $C_{I J K} \geq 0$ and
$Y^{I}(x) \geq 0$ for all $I, J, K$. We will use this positivity repeatedly in what follows.

The assumption that $J$ is contained in a particular, simplicial subcone of a particular Kähler cone may be unreasonable if the Kähler cone is not finitely generated, or if the extended Kähler cone consists of an infinite union of Kähler cones of birationally equivalent Calabi-Yau manifolds. The latter possibility occurs when there is an infinite sequence of flops between isomorphic Calabi-Yau manifolds, and a discrete gauge symmetry is restored at the flop. We expect that our results will carry over to these cases as well, but for the purposes of the present paper we assume that the extended Kähler cone consists of a finite number of finitely generated Kähler cones.

\section{5D SUPERGRAVITY}

Many features of a 5D supergravity are captured by its prepotential, a cubic homogeneous polynomial:

$$
\mathcal{F}:=\frac{1}{6} C_{I J K} Y^{I} Y^{J} Y^{K}:=1 .
$$

In an M-theory compactification to 5D on a Calabi-Yau threefold $X$, indices $I, J, K$ run from 1 to $h^{1,1}(X)$, the constants $C_{I J K}$ are the triple intersection numbers of (2), and the moduli $Y^{I}$ are volumes of calibrated two-cycles; see (4). The constraint $\mathcal{F}:=1$ follows from the fact that the overall volume of the Calabi-Yau is not a vector multiplet modulus in 5D, so the vector multiplet moduli space has dimension $h^{1,1}(X)-1$, and may be thought of geometrically as the $\mathcal{F}=1$ slice of the extended Kähler cone. ${ }^{2}$ At a generic point in moduli space, the gauge group is $U(1)^{h^{1,1}(X)}$, and the gauge kinetic matrix is given by ${ }^{3}$

$$
a_{I J}=\mathcal{F}_{I} \mathcal{F}_{J}-\mathcal{F}_{I J},
$$

with

$$
\mathcal{F}_{I}:=\frac{1}{2} C_{I J K} Y^{J} Y^{K}, \quad \mathcal{F}_{I J}:=C_{I J K} Y^{K} .
$$

The eigenvalues of the gauge kinetic matrix correspond to the inverse squares of gauge couplings, $\lambda_{I} \sim 1 / g_{I}^{2}$. Thus, the eigenvalues of $a_{I J}$ are positive semidefinite everywhere in moduli space and positive definite at a generic points, and an eigenvalue of $a_{I J}$ blows up precisely when a gauge coupling vanishes.

The metric on moduli space is given by the pullback of the gauge kinetic matrix to the $\mathcal{F}=1$ slice of the extended Kähler cone,

\footnotetext{
${ }^{2}$ In $4 \mathrm{D} \mathcal{N}=2$ compactifications of type IIA string theory, there may be additional nongeometric phases in the vector multiplet moduli space, but in 5D these phases are absent [16].

${ }^{3}$ See, e.g., $[17,18]$. We set $2 \kappa_{5}^{2}=1$, with $Y_{\text {(here) }}^{I}=\sqrt{3} h_{\text {(there) }}^{I}$ and $C_{I J K}^{\text {(here) }}=\frac{2}{\sqrt{3}} \mathcal{C}_{I J K}^{\text {(there) }}$.
} 


$$
\left(\frac{d s}{d x}\right)^{2}=a_{I J} \frac{d Y^{I}}{d x} \frac{d Y^{J}}{d x}=\left(\mathcal{F}_{I} \frac{d Y^{I}}{d x}\right)^{2}-\mathcal{F}_{I J} \frac{d Y^{I}}{d x} \frac{d Y^{J}}{d x}
$$

To show that points of zero gauge coupling lie at infinite distance in moduli space, we consider a path in vector multiplet moduli space that approaches a point of zero gauging coupling. As discussed previously, we assume the path in question lies inside a fixed simplicial subcone of a fixed Kähler cone as we approach zero gauge coupling, described by

$$
\forall I, \quad Y^{I} \geq 0,
$$

in an appropriate basis. The intersection numbers are nonnegative in this basis:

$$
\forall I, J, K, \quad C_{I J K} \geq 0 .
$$

The ability to choose a basis where (9) and (10) hold as zero gauge coupling is approached amounts to a regularity condition on the path: we assume it does not meander back and forth indefinitely between different Kähler cones $\mathcal{K}(X), \mathcal{K}(\tilde{X})$ within the extended Kähler cone, nor does it endlessly meander in and out of the simplicial subcone of $\mathcal{K}(X)$ generated by the $\omega_{I}$. This assumption is justified when seeking distance-minimizing paths, as such meandering will only serve to increase the path length.

Per (6), some component of $Y^{I}$ must go to infinity for an eigenvalue of $a_{I J}$ to blow up. Let $Y^{I}=Y^{I}(x)$ be an arbitrary parametrization of the path, with $x=0$ the point at which a gauge coupling goes to zero. We assume a Laurent expansion of the form

$$
Y^{I}(x)=\sum_{n=-N}^{\infty} x^{n} Y_{n}^{I}
$$

for some $N>0$, where the path lies entirely within the cone $Y^{I} \geq 0$ for a finite interval $0<x<x_{0}{ }^{4}$

To show that zero gauge coupling lies at infinite distance, we proceed by contradiction: assuming the path length to be finite, we derive $Y_{n}^{I}=0$ for all $n<0$, and therefore $Y^{I}(x)$ is finite as $x \rightarrow 0$, so $a_{I J}$ is also finite.

\section{A POSITIVITY LEMMA}

We first derive a basic consequence of (9), (10), which we use repeatedly in what follows. While clearly

$$
C_{I J K} Y^{I} Y^{J} Y^{K} \geq 0
$$

\footnotetext{
${ }^{4}$ This ansatz is more general than it appears. For instance, by appropriately redefining $x$, such a Laurent expansion exists for any path specified by algebraic functions $Y^{I}(x)$. (Algebraic functions occur naturally in this context due to the polynomial nature of the prepotential.)
}

in general there is no constraint of the form $C_{I J K} Y_{m}^{I} Y_{n}^{J} Y_{p}^{K} \geq 0$ on the Laurent coefficients. The following lemma establishes when such a constraint holds:

$$
\text { If } C_{I J K} Y_{q}^{I} Y_{r}^{J} Y_{s}^{K}=0 \text { for all }(q, r, s)<(m, n, p)
$$

then $C_{I J K} Y_{m}^{I} Y_{n}^{J} Y_{p}^{K} \geq 0$.

Here we define a partial order on tuples:

$$
(i, j, k) \leq\left(i^{\prime}, j^{\prime}, k^{\prime}\right) \quad \text { if } i \leq i^{\prime}, j \leq j^{\prime}, k \leq k^{\prime},
$$

and $(i, j, k)<\left(i^{\prime}, j^{\prime}, k^{\prime}\right)$ indicates distinct ordered tuples.

To prove (13), let $S_{n}$ be the set of indices $I$ for which the Laurent expansion of $Y^{I}(x)$ has leading term $x^{n}$ (i.e., for which $Y_{n}^{I} \neq 0$ and $\left.Y_{m<n}^{I}=0\right)$. This divides the indices $I$ into disjoint sets $S_{n}, n=-N, \ldots, \infty$. Writing an index $I$ restricted to lie within $S_{n}$ as $I_{n}$, (9) implies

$$
Y_{n}^{I_{n}}>0
$$

as the leading term cannot be negative as $x \rightarrow 0$. Thus:

$$
C_{I J K} Y_{m}^{I} Y_{n}^{J} Y_{p}^{K}=\sum_{(q, r, s) \leq(m, n, p)} C_{I_{q} J_{r} K_{s}} Y_{m}^{I_{q}} Y_{n}^{J_{r}} Y_{p}^{K_{s}}
$$

Next, we show inductively that $C_{I J K} Y_{q}^{I} Y_{r}^{J} Y_{s}^{K}=0$ for $(q, r, s) \leq(m, n, p)$ if and only if $C_{I_{q} J_{r} K_{s}}=0$ for $(q, r, s) \leq(m, n, p)$. Clearly

$$
C_{I J K} Y_{-N}^{I} Y_{-N}^{J} Y_{-N}^{K}=C_{I_{-N} J_{-N} K_{-N}} Y_{-N}^{I_{-N}} Y_{-N}^{J_{-N}} Y_{-N}^{K_{-N}},
$$

which vanishes if and only if $C_{I_{-N} J_{-N} K_{-N}}=0$ because $Y_{-N}^{I_{-N}}>0$. Now suppose that $C_{I J K} Y_{q}^{I} Y_{r}^{J} Y_{s}^{K}=0$ and $C_{I_{q} J_{r} K_{s}}=0$ for all $(q, r, s) \leq(m, n, p)$ for some particular $m, n, p$. We find

$$
C_{I J K} Y_{m+1}^{I} Y_{n}^{J} Y_{p}^{K}=C_{I_{m+1} J_{n} K_{p}} Y_{m+1}^{I_{m+1}} Y_{n}^{J_{n}} Y_{p}^{K_{p}},
$$

which vanishes if and only if $C_{I_{m+1} J_{n} K_{p}}=0$. The same conclusion follows when incrementing $n$ or $p$, completing the proof by induction.

Thus, if $C_{I J K} Y_{q}^{I} Y_{r}^{J} Y_{s}^{K}=0$ for all $(q, r, s)<(m, n, p)$ then $C_{I_{q} J_{r} K_{s}}=0$ for all $(q, r, s)<(m, n, p)$, implying

$$
C_{I J K} Y_{m}^{I} Y_{n}^{J} Y_{p}^{K}=C_{I_{m} J_{n} K_{p}} Y_{m}^{I_{m}} Y_{n}^{J_{n}} Y_{p}^{K_{p}}
$$

This is non-negative per (10), (15), so (13) is proven. 


\section{PROOF OF INFINITE DISTANCE}

Consider the constraint

$$
\frac{1}{6} C_{I J K} Y^{I} Y^{J} Y^{K}=1,
$$

and expand the left-hand side in negative powers of $x$. We show that

$$
C_{I J K} Y_{m}^{I} Y_{n}^{J} Y_{p}^{K}=0 \quad \text { for } m+n+p<0 .
$$

In particular, the leading power of $x$ gives

$$
\frac{1}{6} C_{I J K} Y_{-N}^{I} Y_{-N}^{J} Y_{-N}^{K} x^{-3 N}=0,
$$

therefore $C_{I J K} Y_{-N}^{I} Y_{-N}^{J} Y_{-N}^{K}=0 . \quad$ Next, suppose that $C_{I J K} Y_{m}^{I} Y_{n}^{J} Y_{p}^{K}=0$ for all $m+n+p<-M$, where $M>0$. The leading power of $x$ now gives

$$
\frac{1}{6} \sum_{\substack{m, n, p \\ m+n+p=-M}} C_{I J K} Y_{m}^{I} Y_{n}^{J} Y_{p}^{K} x^{-M}=0 .
$$

The lemma (13) implies that each term in the sum is nonnegative; hence, each term vanishes individually, proving (21) by induction.

The path length is finite if and only if the Laurent expansion of $\left(\frac{d s}{d x}\right)^{2}$ at $x=0$ has no $1 / x^{2}$ (log divergent) or more singular (power-law divergent) term. From (8), we obtain

$$
\begin{aligned}
\left(\frac{d s}{d x}\right)^{2} & =\frac{1}{x^{2}}\left(\frac{1}{2} \sum_{m, n, p} m C_{I J K} Y_{m}^{I} Y_{n}^{J} Y_{p}^{K} x^{m+n+p}\right)^{2}-\frac{1}{x^{2}} \sum_{m, n, p} m n C_{I J K} Y_{m}^{I} Y_{n}^{J} Y_{p}^{K} x^{m+n+p}, \\
& =\frac{1}{x^{2}}\left(\frac{1}{6} \sum_{m, n, p}(m+n+p) C_{I J K} Y_{m}^{I} Y_{n}^{J} Y_{p}^{K} x^{m+n+p}\right)^{2}-\frac{1}{3 x^{2}} \sum_{m, n, p}(m n+m p+n p) C_{I J K} Y_{m}^{I} Y_{n}^{J} Y_{p}^{K} x^{m+n+p} .
\end{aligned}
$$

Per (21), the most singular power that can occur is $1 / x^{2}$. This term takes the form:

$$
\left(\frac{d s}{d x}\right)_{-2}^{2}=\frac{1}{3} \sum_{m, n}\left(m^{2}+m n+n^{2}\right) C_{I J K} Y_{m}^{I} Y_{n}^{J} Y_{-m-n}^{K} .
$$

Each summand is non-negative by (13), therefore

$$
C_{I J K} Y_{m}^{I} Y_{n}^{J} Y_{-m-n}^{K}=0, \quad(m, n) \neq(0,0),
$$

to have a finite path length.

We now make use of the assumption that the gauge kinetic matrix is positive-definite. Consider

$$
V_{t}^{I}:=\sum_{n} t_{n} Y_{n}^{I} x^{n}
$$

for arbitrary coefficients $t_{n}$. We have

$$
\begin{aligned}
V_{t}^{I} V_{t}^{J} a_{I J}= & {\left[\frac{1}{2} \sum_{m, n, p} t_{m} C_{I J K} Y_{m}^{I} Y_{n}^{J} Y_{p}^{K} x^{m+n+p}\right]^{2} } \\
& -\sum_{m, n, p} t_{m} t_{n} C_{I J K} Y_{m}^{I} Y_{n}^{J} Y_{p}^{K} x^{m+n+p} \geq 0
\end{aligned}
$$

for all $0<x<x_{0}$ and arbitrary $t_{p}$.

Using (28), we show inductively that

$$
C_{I J K} Y_{m}^{I} Y_{n}^{J} Y_{p}^{K}=0 \quad \text { when } m<0,
$$

for all $n, p$. To do so, note that this statement is true when $m+n+p \leq 0$ per (21), (26). Now suppose that it is true for $m+n+p<M, M>0$, i.e.,

$$
\begin{aligned}
& C_{I J K} Y_{m}^{I} Y_{n}^{J} Y_{p}^{K}=0, \quad m<0, \quad m+n+p<M, \\
& C_{I J K} Y_{m}^{I} Y_{n}^{J} Y_{p}^{K} \geq 0, \quad m<0, \quad m+n+p=M,
\end{aligned}
$$

where (31) is a consequence of (13). We choose $t_{p}=0$ for $0 \leq p \leq\lfloor M / 2\rfloor$ and $t_{p}>0$ for $p<0$ or $p>\lfloor M / 2\rfloor$. This ensures that

$\frac{1}{2} \sum_{m, n, p} t_{m} C_{I J K} Y_{m}^{I} Y_{n}^{J} Y_{p}^{K} x^{m+n+p}=O\left(x^{M}\right)+O\left(x^{\left\lfloor\frac{M}{2}\right\rfloor+1}\right)$,

$\sum_{m, n, p} t_{m} t_{n} C_{I J K} Y_{m}^{I} Y_{n}^{J} Y_{p}^{K} x^{m+n+p}=O\left(x^{M}\right)+O\left(x^{2\left\lfloor\frac{M}{2}\right\rfloor+2}\right)$,

where the first term on the right-hand side is the leading contribution from terms with either $m, n$, or $p$ negative, and the second is the leading contribution from terms with $m, n$ and $p$ all non-negative. Thus, the leading contribution to $V_{t}^{I} V_{t}^{J} a_{I J}$ is at order $x^{M}$, only the second term in (28) contributes at this order, and only the terms in the sum with either $m, n$, or $p$ negative contribute. Thus,

$$
V_{t}^{I} V_{t}^{J} a_{I J}=-\sum_{m, n} t_{m} t_{n} C_{I J K} Y_{m}^{I} Y_{n}^{J} Y_{M-m-n}^{K} x^{M}+O\left(x^{M+1}\right) .
$$


Each summand is nonpositive per (31), so each must vanish individually. Symmetrizing

$$
\left(t_{m} t_{n}+t_{m} t_{p}+t_{n} t_{p}\right) C_{I J K} Y_{m}^{I} Y_{n}^{J} Y_{p}^{K}=0, \quad m+n+p=M .
$$

Given $m<0$, the constraint $m+n+p=M$ implies that either $n>\lfloor M / 2\rfloor$ or $p>\lfloor M / 2\rfloor$; hence, either $t_{m} t_{n}>0$ or $t_{m} t_{p}>0$, implying $t_{m} t_{n}+t_{m} t_{p}+t_{n} t_{p}>0$. Thus,

$$
C_{I J K} Y_{m}^{I} Y_{n}^{J} Y_{p}^{K}=0, \quad m+n+p=M, \quad m<0,
$$

which establishes (29) by induction.

As a corollary, using (6), (7) we obtain

$$
a_{I J} Y_{n}^{I} Y_{n}^{J}=0, \quad n<0 .
$$

Since $a_{I J}$ is assumed positive definite along the path, this implies $Y_{n}^{I}=0$ for $n<0$; hence, the Laurent expansion is actually a Taylor expansion, and $Y^{I}$ remains finite along the path. This implies that $a_{I J}$ also remains finite, and in particular we cannot reach a zero-coupling point along a finite-length path. This establishes our desired result: points of vanishing gauge coupling lie at infinite distance in moduli space.

\section{PROOF OF ZERO GAUGE COUPLING}

We now show the converse: points at infinite distance in vector multiplet moduli space are necessarily points of vanishing gauge coupling. This has been shown previously in $[11,12]$ using asymptotic Hodge theory [19-22] (see also $[23,24])$, but here we will establish the result using simply the cubic nature of the prepotential and the positivity conditions discussed previously.

It will prove useful to extend our discussion away from the $\mathcal{F}=1$ slice of the Kähler cone, instead letting the $Y^{I}$ be homogenous coordinates invariant under rescaling $Y^{I} \rightarrow \lambda Y^{I}$. $a_{I J}$ extends straightforwardly to the space of homogenous coordinates,

$$
a_{I J}=\frac{\mathcal{F}_{I} \mathcal{F}_{J}}{\mathcal{F}^{4 / 3}}-\frac{\mathcal{F}_{I J}}{\mathcal{F}^{1 / 3}}
$$

Extending the metric requires a little more care. We define projected coordinates

$$
\hat{Y}^{I}=\frac{Y^{I}}{\mathcal{F}^{1 / 3}},
$$

which necessarily satisfy $C_{I J K} \hat{Y}^{I} \hat{Y}^{J} \hat{Y}^{K}=6$. We then have the metric

$$
d s^{2}=a_{\hat{K} \hat{L}}(\hat{Y}) d \hat{Y}^{I} d \hat{Y}^{J}=a_{\hat{K} \hat{L}} \frac{\partial \hat{Y}^{\hat{K}}}{\partial Y^{I}} \frac{\partial \hat{Y}^{\hat{L}}}{\partial Y^{J}} d Y^{I} d Y^{J}
$$

on the space of homogenous coordinates $Y^{I}$, where $a_{\hat{K} \hat{L}}$ is given by (6). Using (38), this can be written as

$$
d s^{2}=g_{I J} d Y^{I} d Y^{J}, \quad g_{I J}=\frac{2}{3} \frac{\mathcal{F}_{I} \mathcal{F}_{J}}{\mathcal{F}^{2}}-\frac{\mathcal{F}_{I J}}{\mathcal{F}} .
$$

This metric is positive-semidefinite: all eigenvalues are positive inside the Kähler cone except for the null eigenvalue corresponding to rescaling $Y^{I} \rightarrow \lambda Y^{I}$.

Next, suppose $Y_{0}^{I}$ is a point at infinite distance in moduli space. We will argue that $Y_{0}^{I}$ is also a point of vanishing gauge coupling. As before, consider a path with endpoint $Y^{I}(x=0)=Y_{0}^{I}$ that is contained entirely within a region $Y^{I} \geq 0$ in a basis where $C_{I J K} \geq 0$. This implies that $\mathcal{F}_{I J}, \mathcal{F}_{I}$, and $\mathcal{F}$ are non-negative for all $I, J$.

By homogeneous rescaling, we can ensure that each $Y^{I}$ remains finite in the limit $Y^{I} \rightarrow Y_{0}^{I}$, and at least one $Y^{L}$ remains nonzero. This coordinate choice implies that $\dot{Y}^{I}$ also remains finite for all $I$, so infinite distance requires at least one eigenvalue of $g_{I J}$ to diverge. Since each $Y^{I}$ is finite, each $\mathcal{F}_{I}$ is also finite, which by (40) means $\mathcal{F}$ must vanish at $Y_{0}^{I}$.

Next, we show that in our chosen coordinate system there exists $K$ such that $\mathcal{F}_{K}^{2} / \mathcal{F}$ is nonzero at the point $Y_{0}^{I}$. Since $Y_{0}^{L}$ is nonzero, there exists at least one pair of indices $K, M$ such that $\mathcal{F}_{K M}=C_{K M L} Y^{L}$ remains nonzero in the limit $Y^{I} \rightarrow Y_{0}^{I}$; otherwise, $a_{I J} Y_{0}^{I} Y_{0}^{J}=0$ everywhere in moduli space, contradicting positive definiteness of $a_{I J}$ in the interior of the Kähler cone. If $K=M$, then $g_{K K} \geq 0$ (required by positive semidefiniteness of $g_{I J}$ ) implies $\left(\mathcal{F}_{K}\right)^{2} / \mathcal{F}$ is nonzero at $Y_{0}^{I}$. If $K \neq M$, then consider

$$
\mathcal{F}^{2} g_{K K} g_{M M} \geq \mathcal{F}^{2}\left|g_{K M}\right|^{2}
$$

which is required by positive semidefiniteness of $g_{I J}$. If the right-hand side of this inequality vanishes at $Y_{0}^{I}$, then because $\mathcal{F}_{K M}$ is nonzero, $\mathcal{F}_{K} \mathcal{F}_{M} / \mathcal{F}$ must also be nonzero. Alternatively, if the right-hand side is nonzero, then the lefthand side must also be nonzero. $g_{K K}$ and $g_{M M}$ must be nonnegative, so from (40) and the fact that $\mathcal{F}_{I J}$ is non-negative in our coordinate system for all $I, J$, we again conclude that $\mathcal{F}_{K} \mathcal{F}_{M} / \mathcal{F}$ is nonzero. This in turn implies that either $\mathcal{F}_{K}^{2} / \mathcal{F}$ or $\mathcal{F}_{M}^{2} / \mathcal{F}$ is nonzero, so without loss of generality we may assume $\left(\mathcal{F}_{K}\right)^{2} / \mathcal{F}$ is nonzero at $Y_{0}^{I}$.

Since $\left(\mathcal{F}_{K}\right)^{2} / \mathcal{F}$ is nonzero and $\mathcal{F} \rightarrow 0$ as $Y^{I} \rightarrow Y_{0}^{I}$, $\left(\mathcal{F}_{K}\right)^{2} / \mathcal{F}^{4 / 3}$ diverges in this limit. From (37), this implies $a_{K K}$ diverges in the limit unless $\mathcal{F}_{K K} / \mathcal{F}^{1 / 3}=$ $\left(\mathcal{F}_{K}\right)^{2} / \mathcal{F}^{4 / 3}+\mathcal{O}(1)$ terms, but in the latter case $g_{K K}$ is negative due to the factor of $2 / 3$ in the first term of (40) relative to (37), contradicting semidefiniteness of $g_{I J}$. We conclude that $a_{K K}$ is infinite at $Y_{0}^{I}$, so $Y_{0}^{I}$ is indeed a point of vanishing gauge coupling. 
In this note, we have shown that in vector multiplet moduli space in 5D supergravity theories arising from M-theory compactifications on a Calabi-Yau threefold, points of vanishing gauge coupling are at infinite distance, and vice versa. We have not shown, however, that such points necessarily exist in any such supergravity theory: it would worthwhile to prove this or to find a counterexample. Note also that our proofs relied heavily on the positivity conditions (9) and (10), which are ensured by the properties of Calabi-Yau geometry discussed previously. We have not found any counterexamples to the statements we have proven by relaxing these conditions. It would be interesting to find such counterexamples or to develop proofs that do not rely on $C_{I J K}$ positivity. Finally, we have concentrated here on ungauged supergravities in five dimensions: it would be interesting to see to what extent these results generalize to gauged supergravities and to other dimensions.

\section{ACKNOWLEDGMENTS}

We thank Callum Brodie, Sergio Cecotti, Matthew Reece, Nathan Seiberg, Cumrun Vafa, Irene Valenzuela, Timo Weigand, and Edward Witten for useful discussions. We thank Matthew Reece for comments on a draft. B. H. was supported by National Science Foundation Grant No. PHY-1914934 during the final stages of this work, and by Perimeter Institute for Theoretical Physics during its inception. Research at Perimeter Institute is supported by the Government of Canada through the Department of Innovation, Science and Economic Development, and by the Province of Ontario through the Ministry of Research, Innovation and Science. T. R. was supported at the Institute for Advanced Study by the Roger Dashen Membership and by NSF Grant No. PHY-1911298. T. R. was supported at the University of California, Berkeley, by NSF Grant No. PHY-1820912, the Simons Foundation, and the Berkeley Center for Theoretical Physics.
[1] H. Ooguri and C. Vafa, On the geometry of the string landscape and the swampland, Nucl. Phys. B766, 21 (2007).

[2] N. Arkani-Hamed, L. Motl, A. Nicolis, and C. Vafa, The String landscape, black holes and gravity as the weakest force, J. High Energy Phys. 06 (2007) 060.

[3] B. Heidenreich, M. Reece, and T. Rudelius, Sharpening the weak gravity conjecture with dimensional reduction, J. High Energy Phys. 02 (2016) 140.

[4] B. Heidenreich, M. Reece, and T. Rudelius, Evidence for a sublattice weak gravity conjecture, J. High Energy Phys. 08 (2017) 025.

[5] S. Andriolo, D. Junghans, T. Noumi, and G. Shiu, A tower weak gravity conjecture from infrared consistency, Fortschr. Phys. 66, 1800020 (2018).

[6] D. Harlow, Wormholes, emergent gauge fields, and the weak gravity conjecture, J. High Energy Phys. 01 (2016) 122.

[7] B. Heidenreich, M. Reece, and T. Rudelius, The weak gravity conjecture and emergence from an ultraviolet cutoff, Eur. Phys. J. C 78, 337 (2018).

[8] T. W. Grimm, E. Palti, and I. Valenzuela, Infinite distances in field space and massless towers of states, J. High Energy Phys. 08 (2018) 143.

[9] B. Heidenreich, M. Reece, and T. Rudelius, Emergence of Weak Coupling at Large Distance in Quantum Gravity, Phys. Rev. Lett. 121, 051601 (2018).

[10] S.-J. Lee, W. Lerche, and T. Weigand, Emergent strings from infinite distance limits, arXiv:1910.01135.

[11] P. Corvilain, T. W. Grimm, and I. Valenzuela, The swampland distance conjecture for Kähler moduli, J. High Energy Phys. 08 (2019) 075.

[12] N. Gendler and I. Valenzuela, Merging the weak gravity and distance conjectures using BPS extremal black holes, J. High Energy Phys. 01 (2021) 176.
[13] S.-J. Lee, W. Lerche, and T. Weigand, Tensionless strings and the weak gravity conjecture, J. High Energy Phys. 10 (2018) 164.

[14] S.-J. Lee, W. Lerche, and T. Weigand, A stringy test of the scalar weak gravity conjecture, Nucl. Phys. B938, 321 (2019).

[15] T. Rudelius, On the possibility of large axion moduli spaces, J. Cosmol. Astropart. Phys. 04 (2015) 049.

[16] E. Witten, Phase transitions in M theory and F theory, Nucl. Phys. B471, 195 (1996).

[17] E. Bergshoeff, S. Cucu, T. de Wit, J. Gheerardyn, S. Vandoren, and A. Van Proeyen, $N=2$ supergravity in five-dimensions revisited, Classical Quantum Gravity 21, 3015 (2004).

[18] E. Lauria and A. Van Proeyen, $\mathcal{N}=2$ Supergravity in $D=4,5$, 6 Dimensions (Springer, New York, 2020), Vol. 966.

[19] W. Schmid, Variation of Hodge structure: The singularities of the period mapping, Inventiones Mathematicae 22, 211 (1973).

[20] E. Cattani, A. Kaplan, and W. Schmid, Degeneration of hodge structures, Ann. Math. 123, 457 (1986).

[21] E. Cattani and A. Kaplan, Polarized mixed hodge structures and the local monodromy of a variation of hodge structure., Inventiones Mathematicae 67, 101 (1982).

[22] M. Kerr, G. J. Pearlstein, and C. Robles, Polarized relations on horizontal SL(2)'s., Doc. Math. 24, 1295 (2019).

[23] T. W. Grimm, C. Li, and E. Palti, Infinite distance networks in field space and charge orbits, J. High Energy Phys. 03 (2019) 016.

[24] T. W. Grimm, F. Ruehle, and D. van de Heisteeg, Classifying Calabi-Yau threefolds using infinite distance limits, Commun. Math. Phys. 382, 239 (2021). 